DEVELOPMENTAL BIOLOGY

\section{The mole genome reveals regulatory rearrangements associated with adaptive intersexuality}

\author{
Francisca M. Real ${ }^{1,2}$, Stefan A. Haas ${ }^{3}$, Paolo Franchini ${ }^{4}$, Peiwen Xiong ${ }^{4}$, Oleg Simakov ${ }^{5}$, Heiner Kuhl ${ }^{6}$, \\ Robert Schöpflin ${ }^{1,2}$, David Heller ${ }^{3}$, M-Hossein Moeinzadeh ${ }^{3}$, Verena Heinrich ${ }^{3}$, Thomas Krannich ${ }^{3}$, \\ Annkatrin Bressin ${ }^{3}$, Michaela F. Hartmann7, Stefan A. Wudy ${ }^{7}$, Dina K. N. Dechmann ${ }^{8,9}$, \\ Alicia Hurtado $^{10,11}$, Francisco J. Barrionuevo ${ }^{10,11}$, Magdalena Schindler ${ }^{1,2}$, Izabela Harabula ${ }^{1}$ \\ Marco Osterwalder ${ }^{12,13}$, Michael Hiller ${ }^{14,15,16}$, Lars Wittler ${ }^{17}$, Axel Visel ${ }^{12,18,19}$, Bernd Timmermann ${ }^{1}$, \\ Axel Meyer ${ }^{4}$, Martin Vingron ${ }^{3}$, Rafael Jiménez ${ }^{10,11}$, Stefan Mundlos ${ }^{1,2,20 *}$, Darío G. Lupiáñez $z^{1,2,20,21 *}$
}

Linking genomic variation to phenotypical traits remains a major challenge in evolutionary genetics. In this study, we use phylogenomic strategies to investigate a distinctive trait among mammals: the development of masculinizing ovotestes in female moles. By combining a chromosome-scale genome assembly of the Iberian mole, Talpa occidentalis, with transcriptomic, epigenetic, and chromatin interaction datasets, we identify rearrangements altering the regulatory landscape of genes with distinct gonadal expression patterns. These include a tandem triplication involving CYP17A1, a gene controlling androgen synthesis, and an intrachromosomal inversion involving the pro-testicular growth factor gene FGF9, which is heterochronically expressed in mole ovotestes. Transgenic mice with a knock-in mole CYP17A1 enhancer or overexpressing FGF9 showed phenotypes recapitulating mole sexual features. Our results highlight how integrative genomic approaches can reveal the phenotypic impact of noncoding sequence changes.

】 ifferences in genome sequence and structure provide the molecular foundation for phenotypic diversity across species and enable environmental adaptation. In evolutionary genetics, linking genomic alterations to phenotypic traits has largely relied on candidate gene (I) or linkagemapping analyses (2). However, the combination of next-generation sequencing with proximity-ligation methods, in particular Hi-C, allows the generation of chromosome-scale genome assemblies (3), introducing ample possibilities for comparative genomics. Hi-C also enables the integration of three-dimensional (3D) genome structure with transcriptional control. Vertebrate genomes are spatially organized into regulatory units, termed topologically associating domains (TADs) $(4,5)$. Although TADs are generally preserved across species $(6,7)$, studies of human disease highlighted that alterations in TAD organization can cause changes in gene expression and developmental phenotypes by rewiring enhancerpromoter contacts $(8-10)$. Thus, analytical strategies that consider $3 \mathrm{D}$ organization and regulatory potential become essential for a comprehensive annotation of genomes. Here, we introduce a phylogenomic strategy that combines comparative whole-genome, epigenomic, transcriptomic, and chromatin interaction data to identify phenotype-associated genomic changes. We demonstrate the power of this approach by elucidating the molecular underpinnings of generalized intersexuality in female moles, an evolutionary trait distinctive among mammals.

In mammals, sex is determined genetically. Genetic elements direct the differentiation of the bipotential gonad into either testicular or ovarian tissue, which, in turn, leads to the development of sex-specific anatomical, hormonal, and behavioral differences (11). An exception to this paradigm occurs in moles (family Talpidae), in which XX-genotypic females have an intersex phenotype in at least eight species $(12,13)$. Although male moles have normal testes, genotypic females develop ovotestes instead of ovaries (Fig. 1A). These unusual gonads are composed of an ovarian part (OP) that fully supports sexual reproduc- tion and a testicular part (TP) that lacks fertile germ cells but contains typical male cell populations, such as androgen-producing Leydig cells (12) (fig. S1). As a consequence of increased androgen synthesis, female moles develop masculinized external genitalia, as well as prominent muscles and aggressive behavior (14), traits that likely represent adaptations to a subterranean lifestyle.

\section{The Iberian mole genome}

To investigate the molecular origins of mole ovotestes, we generated a chromosome-scale genome assembly for Talpa occidentalis based on long- and short-read sequencing and scaffolded using Hi-C data (Fig. $1 \mathrm{~B}$ and fig. S2). The assembly comprises 2.099 gigabases, and up to $30 \%$ is made of transposable elements, with a repeat profile that differs from that of closely related mammals (fig. S3 and supplementary text). Combining RNA sequencing (RNA-seq) datasets and homology-based predictions, we identified 18,751 genes, including 2370 single-copy orthologs. This gene subset was used to determine 1580 one-toone orthologous genes in nine species and build a phylogenetic tree (Fig. 1C). Our analysis confirms moles as a distinct family in the order Eulipotyphla, with shrews and hedgehogs being the most closely related species (15) (supplementary text).

\section{Epigenetic and transcriptional landscape of mole gonadal development}

We generated epigenetic and transcriptomic profiles of mole gonads at 7 days postpartum (P7), processing TPs and OPs separately (fig. S2). Specifically, we produced chromatin immunoprecipitation sequencing (ChIP-seq) datasets against histone modifications (H3K4me1, H3K4me3, H3K27ac, and H3K27me3) to segment the mole genome into functional states for each tissue. Additionally, we performed assays for transposase-accessible chromatin using sequencing (ATAC-seq) (fig. S2) and intersected both datasets to predict active enhancers in each tissue (22,105 in total) (Fig. 1D and data S1). Although the TP and testis shared a higher number of putative enhancers than the TP and OP, the large number of TP-specific putative

${ }^{1}$ RG Development \& Disease, Max Planck Institute for Molecular Genetics, Berlin, Germany. ${ }^{2}$ Institute for Medical and Human Genetics, Charité - Universitätsmedizin Berlin, Berlin, Germany.

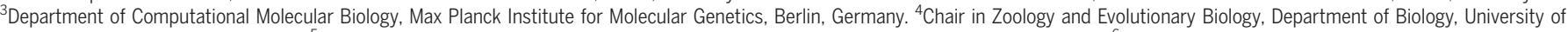
Konstanz, 78457 Konstanz, Germany. ${ }^{5}$ Department of Molecular Evolution and Development, University of Vienna, 1090 Vienna, Austria. ${ }^{6}$ Department of Ecophysiology and Aquaculture, Leibniz-Institute of Freshwater Ecology and Inland Fisheries, Berlin, Germany. ${ }^{7}$ Steroid Research \& Mass Spectrometry Unit, Laboratory for Translational Hormone Analytics in Paediatric

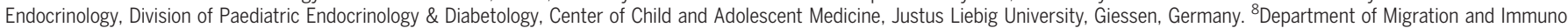
Ecology, Max Planck Institute for Animal Behavior, Radolfzell, Germany. ${ }^{9}$ Department of Biology, University of Konstanz, Konstanz, Germany. ${ }^{10}$ Departamento de Genética, Universidad de Granada, Granada, Spain. ${ }^{11}$ Instituto de Biotecnología, Centro de Investigación Biomédica, Universidad de Granada, Armilla, Granada, Spain. ${ }^{12}$ Environmental Genomics and Systems Biology Division, Lawrence Berkeley National Laboratory, Berkeley, California 94720, USA. ${ }^{13}$ Department for BioMedical Research (DBMR), University of Bern, 3008 Bern, Switzerland. ${ }^{14}$ Max Planck

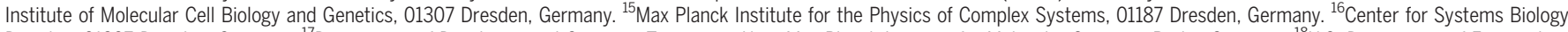

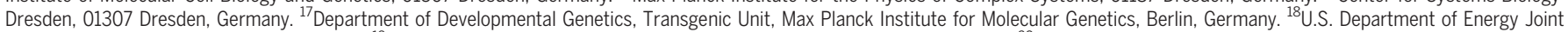

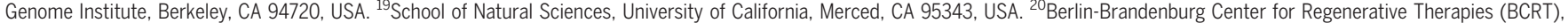
Charité - Universitätsmedizin Berlin, Berlin, Germany. ${ }^{21}$ Epigenetics and Sex Development Group, Berlin Institute for Medical Systems Biology, Max-Delbrück Center for Molecular Medicine, Berlin, Germany.

*Corresponding author. Email: mundlos@molgen.mpg.de (S.M.); dario.lupianez@mdc-berlin.de (D.G.L.) 


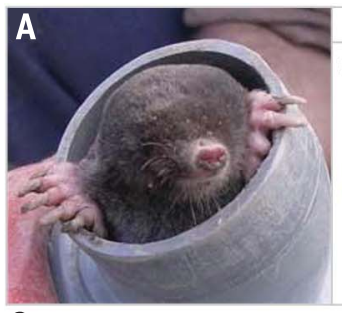

XX Adult Ovotestis

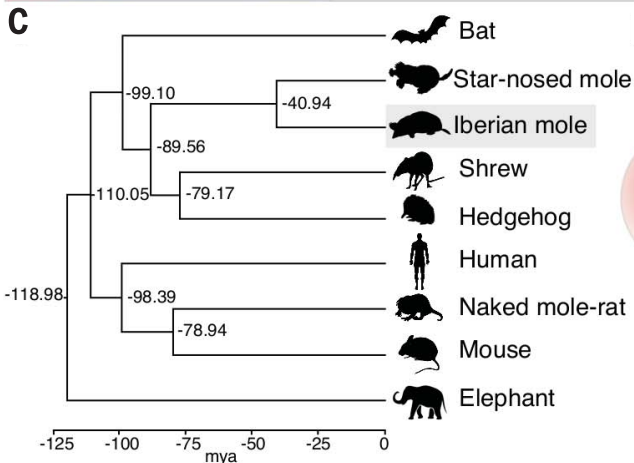

Testis part

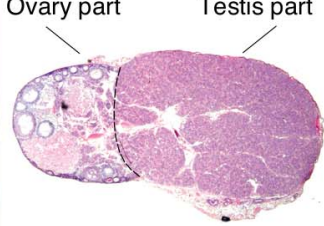

B

\begin{tabular}{|rrr}
\multicolumn{3}{|c}{ Contigs (PacBio) Scaffolds (HiC) } \\
\hline num contigs & 8,093 & 4,454 \\
Largest contig & $21,251,606$ & $155,848,725$ \\
Total length & $2,098,179,395$ & $2,099,999,819$ \\
N50 & $2,611,711$ & $119,847,213$ \\
GC $(\%)$ & 41.91 & 41.91 \\
\hline Annotated genes & & 18,751 \\
Transcripts & & 25,926 \\
\hline
\end{tabular}

\section{D}

\section{Active regulatory} regions

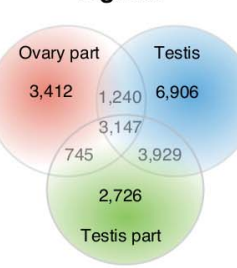

E

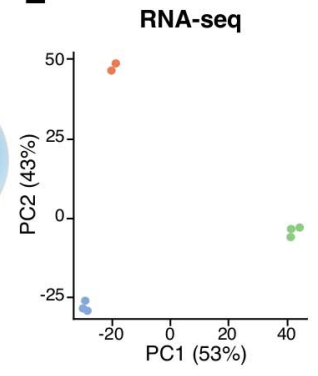

A

Identification of genes with altered 3D chromatin organization

Step 1: Rearrangement detection

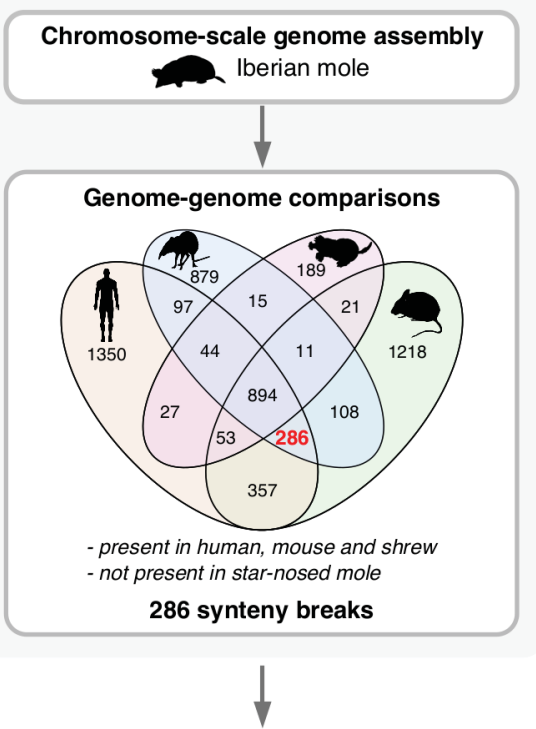

Step 2: Gene identification

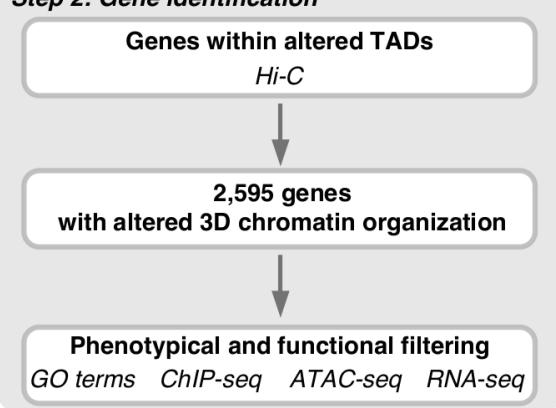

B
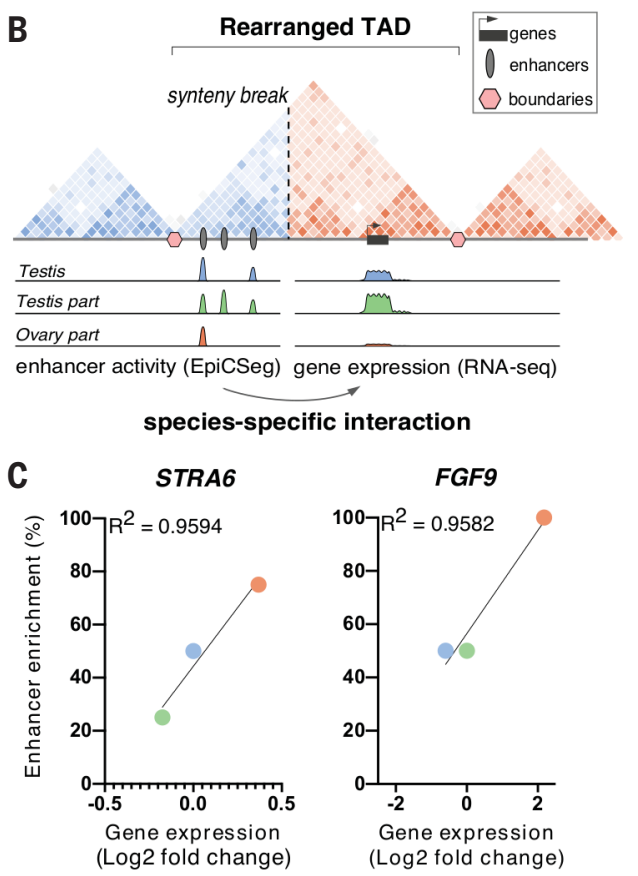

CYP17A1

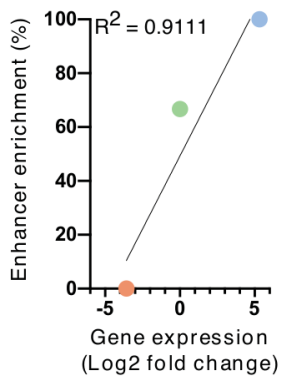

Fig. 1. Mole genome and epigenetic and transcriptional study of ovotestis development. (A) An Iberian mole (Talpa occidentalis) and an adult mole ovotestis. Scale bar, $500 \mu \mathrm{m}$.

(B) Genome assembly of $T$. occidentalis and gene annotation statistics. (C) Phylogenetic tree, based on fourfold degenerate sites, between selected species. mya, million years ago.

(D) Venn diagram of active enhancers (data S1).

(E) Principal component (PC) analysis of RNA-seq datasets of P7 mole gonads.
Fig. 2. Identification of genes with altered 3D chromatin regulatory landscapes.

(A) Strategy used to identify genes with altered 3D chromatin organization as a result of species-specific rearrangements. (B) Strategy used to assign regulatory elements to candidate genes. Number of active enhancers is correlated to gene expression levels for each tissue.

(C) Correlation between the percentage of active enhancers and gene expression per tissue (orange, ovary part; green, testis part; blue, male testis) for selected candidates (full gene dataset in fig. S4 and data S7). STRA6, FGF9, and CYP17A1 display the highest positive correlation; ATM shows negative correlation. 
A

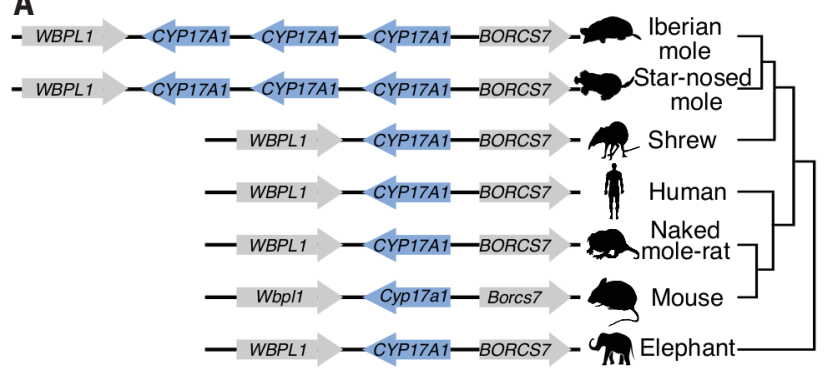

C

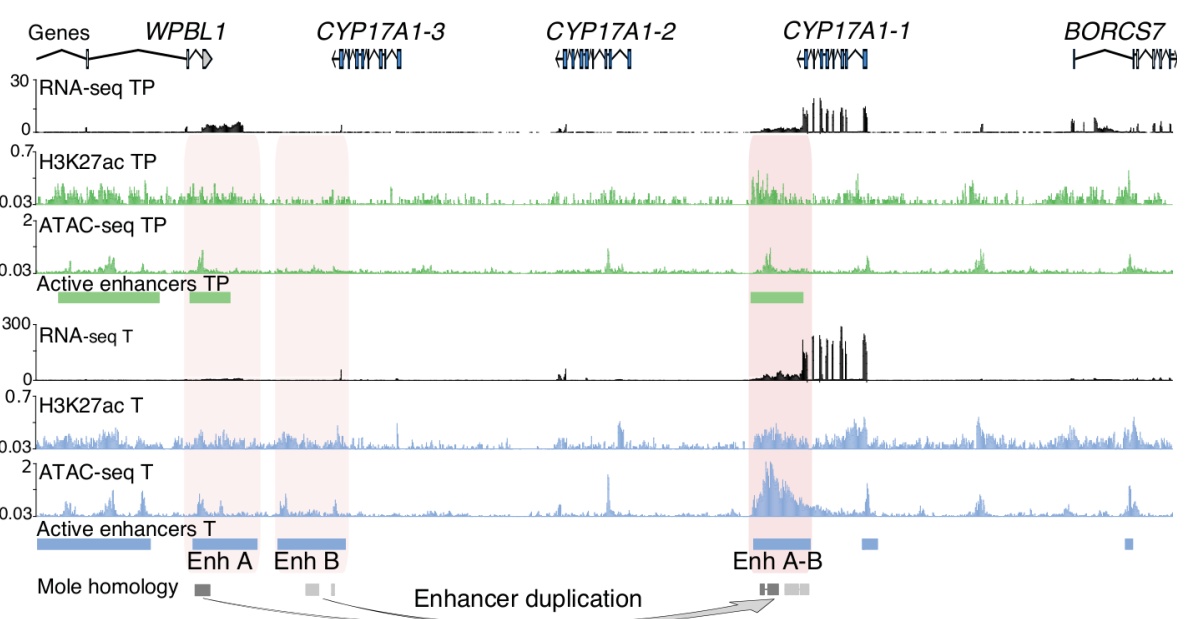

D Knock-in

mole Enh A-B

-WBPL1 - A-B CYP17A1-
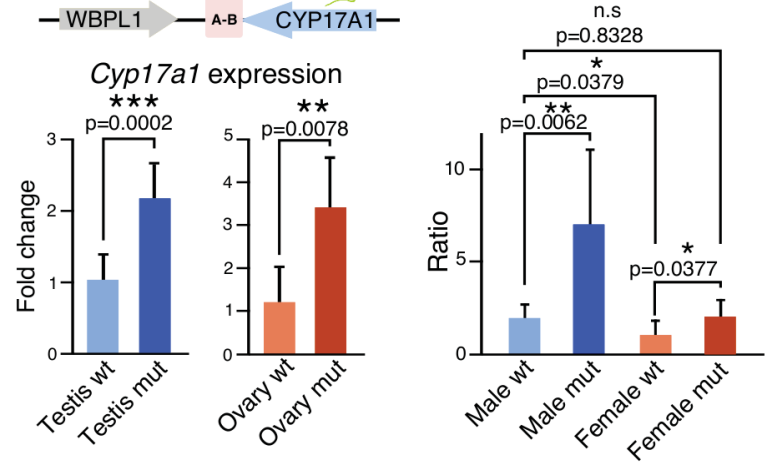

$\mathbf{F}$
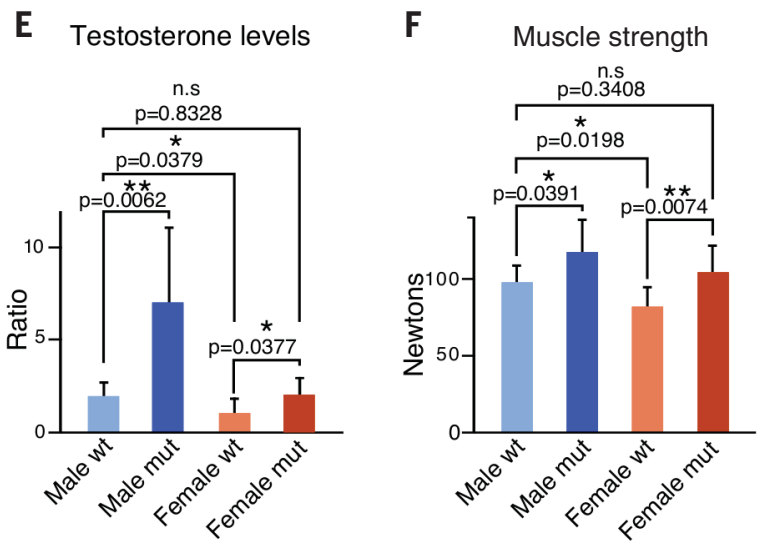

Fig. 3. Duplication of regulatory elements at the CYP17A1 locus and associated increase in androgen production and strength. (A) Comparative genomics at the CYP17A1 locus. (B) CYP17A1 expression (RNA-seq) in mole and mouse adult gonads $(n=2)$. RPKM, reads per kilobase per million reads. (C) Expression profile (RNA-seq, top), enhancer marks (H3K27Ac, center), and open chromatin (ATAC-seq, bottom) for testis part and testis at P7 gonads. Segmentation for active enhancers for testis part (green bars) and testis (blue bars). BLAT Sequence homology is represented in gray boxes. Duplicated enhancer (A-B) results from fusion of enhancer A and B. (D) (Above) Integration of the mole CYP17A1 duplicated enhancer (Enh) into the mouse Cyp17a1 locus. (Below) Expression analysis of Cyp17al (reverse transcription quantitative polymerase chain reaction) in adult mouse mutant gonads (mut) and wild-type controls (wt) $(n \geq 5)$. (E) Circulating testosterone levels in adult mouse mutants and wild-type controls $(n=7)$. (F) Grip-strength test in adult mouse mutants and wild-type controls $(n=7)$. Bars represent mean and SD. Two-sided Student's $t$ test. n.s, nonsignificant. ${ }^{*} P \leq 0.05,{ }^{* *} P \leq 0.01,{ }^{* * *} P \leq 0.001$.

enhancers (2726) indicates a distinct molecular profile from the testis and OP. Principal component analysis of RNA-seq data further confirmed the distinctive nature of the TP (Fig. 1E).

\section{An analytical framework for evolutionary analyses}

We combined our functional datasets with comparative genomics analyses and focused at three distinct levels: genes, regulatory ele- ments, and 3D chromatin organization (fig. S4). We reasoned that phenotype-relevant mutations affecting these levels should be shared by the Iberian mole and the American star-nosed mole (Condylura cristata), whose females also develop ovotestes. We first searched for gene families that underwent expansion or contraction in the mole lineage, as well as genes under positive selection (supplementary text and data S2 and S3). Gene Ontology (GO) enrichment analyses revealed signatures in metabolic, immunological processes and the olfactory receptor repertoire. By filtering with GO terms related to sex differentiation ("sex, gonad") (data S4), we identified eight positively selected genes that could affect mole gonadal development (table S1). To gain functional insight into these genes, we searched for mouse- and human-reported mutations. Although some mutations led to reduced fertility, none was reported to induce sex reversal, thus making a contribution to the mole intersex phenotype unlikely (data S3).

Next, we focused on regulatory elements by identifying mole-accelerated regions, defined as genomic segments that are highly conserved during mammalian evolution but diverged in moles (supplementary text and data S5). We identified 3560 mole-accelerated regions that were subsequently filtered for overlap with our predicted gonadal enhancers (129 regions). Instead of associating the accelerated enhancers to the nearest gene, we used $\mathrm{TAD}$ predictions from Hi-C data to delimit a genomic range of interaction for each element and assign them to the genes in such regions. TADs are well conserved across tissues (fig. S5), consistent with previous findings (4). The assigned genes were further filtered with GO terms related to sex differentiation, which revealed two candidates: the transcription factor (TF) Osr1 and the cell-cycle regulator $C d k 2$ (table S1). Although both genes are essential for gonadogenesis $(16,17)$, they show a similar expression pattern in moles and mice and are likely not relevant for mole ovotestis formation (fig. S6).

Because no relevant candidates were found, we searched for changes in $3 \mathrm{D}$ chromatin organization, on the basis of the hypothesis that rearrangements can alter regulatory domains and affect gene expression $(8,9)$. Rearrangements can be identified in genome comparisons as synteny breaks, defined here as alterations on the conserved colinear order of loci between species. To identify molespecific rearrangements, we compared the mole genomes (T. occidentalis and C. cristata) with full-chromosome assemblies from human, mouse, and shrew, as the closest taxonomical outgroup with normal ovarian development (Fig. 2A). We used our Hi-C domain predictions to identify genes located in TADs affected by a synteny break, for a total of 2595 candidate 
genes considered to be susceptible to altered regulation (data $\mathrm{S6}$ ). We filtered these candidates according to GO terms related to sex differentiation, restricting the list to 39 genes (table S1 and data S4).

Using our functional datasets, we then searched for footprints of altered gene regulation that might be the consequence of molespecific rearrangements. We considered the nature of each rearrangement (Fig. 2B and supplementary text) and our TAD predictions, to delimit a potential region of new interactions for each candidate gene and determine the number of active regulatory elements contained within. Of the 39 candidate genes, only 17 were predicted to gain de novo interaction with regions containing active enhancers. Furthermore, we ranked the candidate genes by correlating the number of active elements with the expression levels for each tissue, as an indicative parameter of potential effects of the rearrangement on transcription (Fig. $2 \mathrm{C}$, fig. S7, and data S7). A positive correlation between active enhancers and gene expression was found for 10 genes. Among the top-ranked candidates, we selected those displaying higher expression for subsequent functional validation: the androgen-related gene CYP17A1 and the pro-testicular growth factor gene FGF9.

\section{A tandem triplication at the CYP17A1 locus is linked to increased androgen production and strength}

We detected an intra-TAD tandem triplication at the mole CYP17A1 locus that creates two additional copies of the gene. Through comparative genomics analysis, we confirmed the exclusive presence of the rearrangement in the mole lineage and its absence in other mammals (Fig. 3A and fig. S8). CYP17A1 encodes a key enzyme controlling androgen synthesis (18), suggesting a role in female mole masculinization. The triplication was associated with high CYP17A1 expression in testis and TP, both substantially exceeding the expression levels in mice (Fig. 3B and data S8). Searching for other genes of the steroidogenic pathway, we observed that CYP19A1, located downstream of CYP17A1, is not expressed in the TP (fig. S9). CYP19A1 encodes for aromatase, an enzyme that converts androgens to estrogens (18), and is expressed exclusively in the OP (fig. S9). It is thus expected that the high levels of CYP17A1 in the OP do not impede estrogen production and reproductive function, because of the protective effect of aromatase in degrading androgens locally. The absence of CYP19A1 expression in the TP, in combination with high CYP17A1 expression, provides a plausible explanation for the masculinization observed in female moles. We used gas chromatography-mass spectrometry to quantify serum levels of male hormones and found high levels of circulating

A
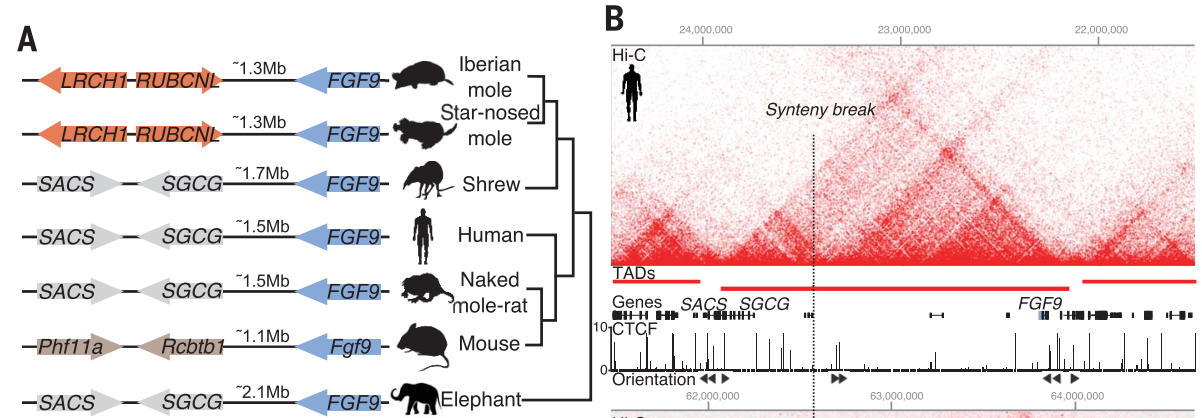

C
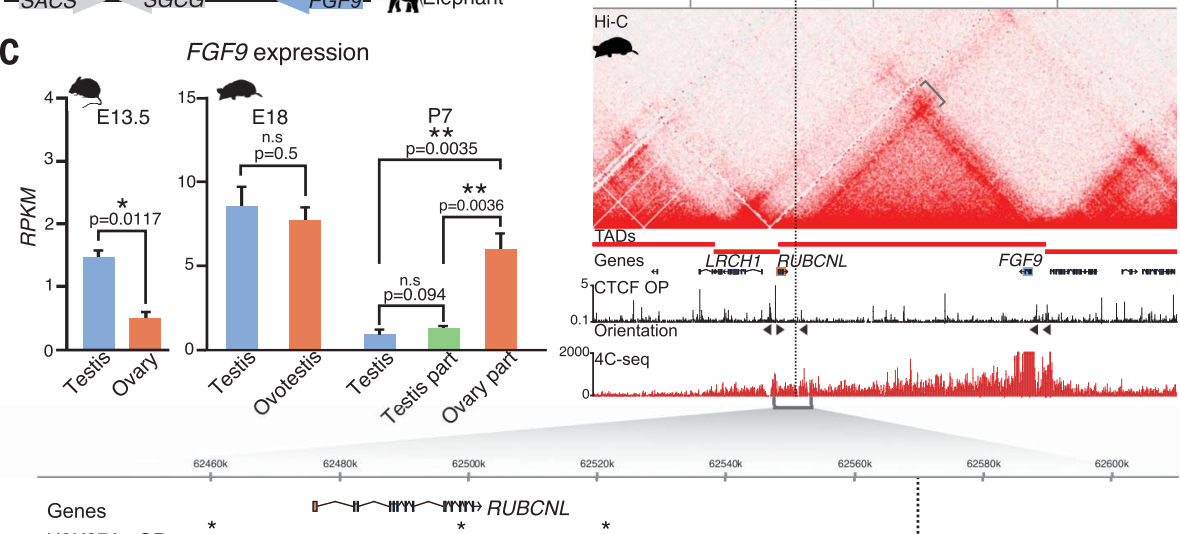

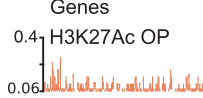

$\left.{ }^{2}\right]$ ATAC-seq OP
Active enhancers OP

${ }^{5}$ ] CTCF OP

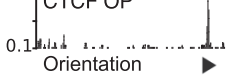

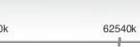

62560k

$62580 \mathrm{k}$ 62600k

g. 4. An inversion altering the regulatory landscape of the FGF9 mole locus. (A) Comparative genomics at the FGF9 locus. (B) Hi-C maps for human and mole displaying synteny break (discontinuous line) and TAD prediction. (Below) CTCF ChIP-seq (mole P7 gonads) with peak orientation. 4C-seq using mole FGF9 promoter as viewpoint shows contact extension beyond synteny break. Zoom of FGF9 interacting region shows active ovarian enhancers (asterisks). H3K27Ac, ATAC-seq, and segmentation for active enhancer tracks are displayed in orange. (C) FGF9 expression (RNA-seq) in mice and moles at different time points. Bars represent mean and SD $(n \geq 2)$. Two-sided Student's $t$ test. n.s $=$ nonsignificant. ${ }^{*} P \leq 0.05$, ${ }^{* *} P \leq 0.01$.

androgens in female moles. Androgen levels were similar to those in male individuals (fig. S9), contrary to the general pattern among mammals in which males display higher levels than females.

To examine the contributions of the additional CYP17A1 alleles to increased androgen production, we analyzed RNA-seq data from mole testes and TP. The three CYP17A1 paralogues have sufficiently diverged to enable unambiguous mapping of RNA-seq reads. The two newly emerged CYP17A1-2 and CYP17A1-3 jointly contribute less than $5 \%$ of the CYPI7A1 transcript (Fig. 3C). Furthermore, sequence conservation analyses revealed that they diverge more from the human sequence than CYP17A1-1 (fig. S10). These findings suggest that the triplication of the CYP17A1 gene itself does not explain the increased androgen levels in moles. Instead, the triplication also caused the duplication and fusion of two predicted enhancer elements, termed "enhancer A" and "enhancer B" (Fig. 3C). As the triplication does not affect any TAD boundary, the duplicated enhancers and CYP17A1 genes locate within the same regulatory domain (fig. S11). The duplicated fusion element, "enhancer A-B," shows a high degree of sequence conservation with the original elements ( 84 and $90 \%$, respectively) and high abundance of active enhancer marks (Fig. 3C). Computational predictions of binding affinities showed that enhancer A-B maintains significant binding affinities for TFs found in the original enhancer A, as well as previously uncharacterized binding affinities for TFs that are expressed in the TP (data S9). Together, these observations suggest that duplication and functional changes in regulatory sequences, rather than amplification of coding sequence, cause the observed phenotypic adaptations.

To confirm this hypothesis, we inserted the mole enhancer A-B sequence into the Cyp17a1 

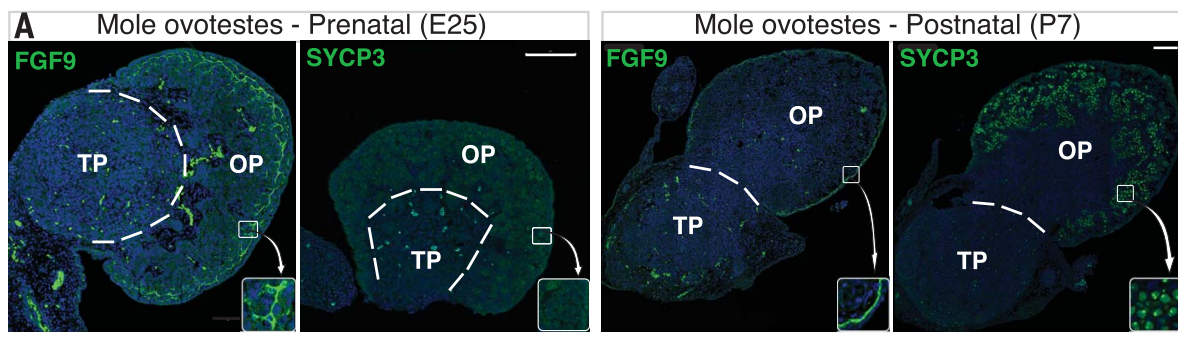

B

RNA-seq E13.5 Fgf9 mouse overexpressing ovaries

\begin{tabular}{ll}
\multicolumn{1}{c}{ GO terms (down) } & p-value \\
\hline meiotic cell cycle & $2.93 \mathrm{E}-29$ \\
female gamete generation & $1.26 \mathrm{E}-11$
\end{tabular}

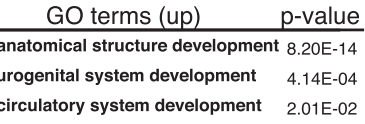
.Col6a3
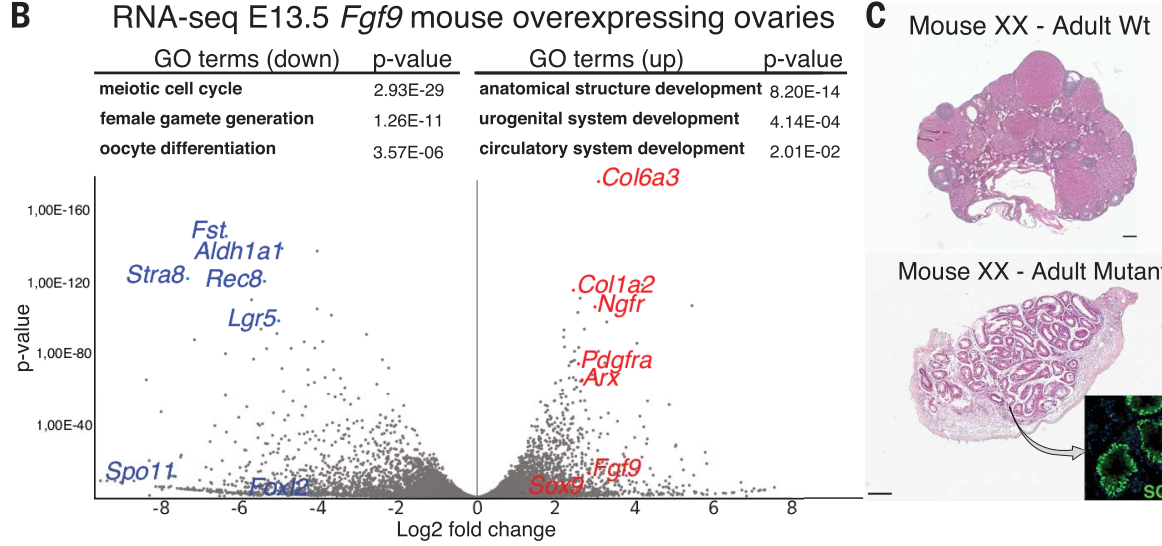

Mouse XX - Adult Mutant

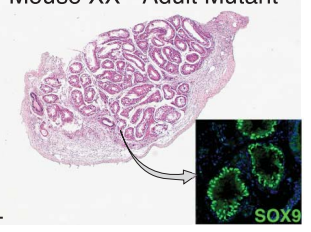

Fig. 5. FGF9 sustained expression delays meiosis and promotes $X X$ gonadal masculinization.

(A) Spatiotemporal expression of FGF9 and the meiotic marker SYCP3 [immunostaining, green; 4',6-diamidino-2-phenylindole (DAPI), blue]. Insets display zoomed regions from OP. Scale bars, $100 \mu \mathrm{m}$. (B) Volcano plot from RNA-seq of XX mutant versus wild-type gonads (E13.5) and G0 analysis. (C) Hematoxylin and eosin staining of XX gonads of adult mutants and wild-type controls. Cord-like structures in mutants denote $X X$-to-XY sex reversal. Inset shows SOX9 expression (immunostaining, green; DAPI, blue). Scale bars, $200 \mu \mathrm{m}$.

murine locus (Fig. 3D). Adult knock-in mice carrying the additional mole enhancer displayed a three- and twofold up-regulation of Cyp17a1 expression in females and males, respectively. The increased expression occurs in the same cell type (steroidogenic cells) as in wild-type controls (fig. S12). A similar effect was observed at embryonic stages (fig. S12), thus confirming the increased in vivo activity of the mole-specific fusion enhancer in gonadal tissue. The up-regulation of Cyp17a1 expression was accompanied by an increase in circulating testosterone in females and males (two- and threefold respectively) (Fig. 3E). Because androgens have potent anabolic effects in muscle, we performed a grip-strength test that revealed a significant increase in muscle strength in mutants compared with wild-type controls (Fig. 3F). Thus, the regulatory nature of the CYP17A1 rearrangement offers a plausible molecular mechanism for the observed shift in hormone levels and the corresponding phenotype.

\section{An inversion at the FGF9 locus is associated with delayed meiosis and gonadal masculinization}

We identified a rearrangement at the FGF9 locus, which is exclusive to the mole lineage and not present in any other mammals exam- ined (Fig. 4A and figs. S13 and S14). FGF9 is a known testis-determining gene that functions in positive feedback with $S O X 9$ and inhibits the ovary-determining $W N T 4 / \beta$-catenin pathway (11) (fig. S15). Consequently, loss of $F g f 9$ in XY gonads results in down-regulation of testicular markers and male-to-female sex reversal. Comparative analyses against human, representative of the ancestral organization at the locus, revealed a large inversion that relocates a distant genomic region $(26 \mathrm{Mb}$ away in the human genome) to the mole FGF9 locus (Fig. 4B). Hi-C data showed that the synteny break occurs in the human FGF9 TAD, disrupting its $3 \mathrm{D}$ organization. Thus, the mole locus is reorganized, with most of the FGF9 TAD remaining conserved but extending beyond the synteny breakpoint on the centromeric side. This extended interaction domain is delimited by the presence of two CTCF binding sites with divergent orientation, a genomic signature associated with TAD boundaries (7) (Fig. 4B). A closer examination of the newly interacting region revealed several elements enriched for active epigenetic marks, some of them specific for the mole ovotestis (fig. S16). This interaction pattern was also validated through independent circular chromosome conformation capture sequencing (4C-seq) experiments (Fig. 4B), which con- firmed the association of the FGF9 gene with the region containing the putative tissue-specific enhancers. We tested one element (fig. S16) in mouse LacZ reporter assays. Although this element displayed enhancer activity in tissues such as the eye (fig. S17), we did not observe gonadal staining, which could be due to specific requirements for additional trans-acting factors in the mole. Alternatively, it may reflect known limitations of the reporter assay that might be intensified by the interspecies nature of the experiment (19).

We then explored possible alterations on the dimorphic FGF9 expression pattern observed in other mammals, which is essential for suppressing germ cell meiosis (20). In mice, $F g f g$ is first expressed in the bipotential gonad of both sexes and becomes progressively restricted to the testis and turned off in ovaries, allowing the initiation of female meiosis at embryonic day 13.5 (E13.5). In mole gonads, however, FGF9 expression is maintained after sex determination in both sexes and becomes restricted to the $\mathrm{OP}$ at later stages (Fig. 4C). Immunostaining analyses showed that FGF9 expression persists in the $\mathrm{OP}$ across the entire prenatal period and becomes confined to a thin rim at postnatal stages (P7). The spatial reduction in FGF9 expression is concomitant with the initiation of meiosis (Fig. 5A and fig. S18), which is considerably delayed in female moles until birth, an exceptional feature among mammals (21). Consequently, the observed heterochrony on mole FGF9 expression, compared with mouse, and its potential effects are suggestive of a contribution to the masculinization of female mole gonads.

We hypothesized that FGF9 expression during early XX mole gonadogenesis might prevent germ cells from entering meiosis in the OP, allowing the TP to develop further. To test this hypothesis, we engineered a bacterial artificial chromosome (BAC) construct to overexpress $\mathrm{Fg} f 9$ in somatic ovarian populations and generated transgenic mice through PiggyBac transgenesis and morula aggregation. Highly chimeric animals displayed early embryonic lethality, likely due to $\mathrm{Fg} f 9$ misexpression in other organs, an effect that precluded their study in later stages. Nevertheless, RNA-seq analysis of E13.5 ovaries demonstrated an inhibition of the meiotic process, manifested by downregulation of meiosis markers (Fig. $5 \mathrm{~B}$ and data S10). By contrast, low-chimera individuals composed of XX wild-type and XX mutant cells were viable and displayed $F g f g$ expression during the entire ovarian development. These animals showed a complete female-to-male sex reversal, defined by gonadal morphology and expression of male-specific factors such as SOX9 (Fig. 5C). These results directly confirm the potential of altered FGF9 expression to induce masculinization in mammalian $\mathrm{XX}$ females. 


\section{Discussion}

Vertebrate sex determination is controlled by a limited set of key regulators whose hierarchy has evolved dynamically (11). Most of these genes display pleiotropic effects, controlling regulatory networks in several tissues, often making them indispensable for embryonic viability $(22,23)$. It is thus plausible that variations in sex determination derive from regulatory changes that alter gene expression patterns but preserve essential functions, as suggested for other evolutionary adaptations $(1,2)$. These genomic changes appear to be linked to the evolutionary success of moles and demonstrate that regulatory innovation can overcome a priori seemingly incompatible situations such as female fertility in the presence of high androgen levels. But how could an intersex phenotype evolve, and what is its adaptive significance? One potential explanation is the anabolic effects of androgens on muscle mass. Mole ovotestes may have evolved to equalize muscular strength among sexes by increasing androgens in females. Such an advantage, combined with other androgen-derived effects such as aggressive behavior, might have been key for the adaptation to the demanding requirements of a burrowing underground lifestyle (14).

This study also highlights the evolutionary importance of genomic rearrangements and their potential to modulate developmental gene expression. In most cases, genomic rearrangements would have limited effects, as they preserve whole regulatory units and do not disrupt enhancer-promoter interactions $(6,24)$. However, as shown here, they can also alter the regulatory potential in the local environment of a TAD, as for the CYP17A1 locus, or shuffle the functional content of distant TAD units, exemplified by the FGF9 locus. Similar effects have been observed in human genetic diseases for enhancer duplications (25) and for inversions $(8,9)$. Therefore, regulation of genes by enhancer elements and their organization in
TADs might constitute a mechanism of "evolvability" resulting from a modular system with vast flexibility and enormous evolutionary potential for the origin of novelties. On the basis of this modularity, genomic rearrangements can easily change and reconstitute complex expression patterns, thereby contributing to the saltatory nature of phenotypic innovation observed in many phylogenetic lineages. We expect that approaches considering these important aspects will eventually reveal the evolutionary basis of many other traits and substantially enhance the toolbox for unlocking the secrets of phenotypic variation and adaptation across the animal kingdom.

\section{REFERENCES AND NOTES}

1. J. Lopez-Rios et al., Nature 511, 46-51 (2014).

2. Y. F. Chan et al., Science 327, 302-305 (2010).

3. 0. Dudchenko et al., Science 356, 92-95 (2017).

4. J. R. Dixon et al., Nature 485, 376-380 (2012).

5. E. P. Nora et al., Nature 485, 381-385 (2012).

6. N. Harmston et al., Nat. Commun. 8, 441 (2017).

7. C. Gómez-Marín et al., Proc. Natl. Acad. Sci. U.S.A. 112 , 7542-7547 (2015).

8. M. Spielmann, D. G. Lupiáñez, S. Mundlos, Nat. Rev. Genet. 19, 453-467 (2018)

9. D. G. Lupiáñez et al., Cell 161, 1012-1025 (2015)

10. M. Franke et al., Nature 538, 265-269 (2016).

11. B. Capel, Nat. Rev. Genet. 18, 675-689 (2017)

12. F. J. Barrionuevo, F. Zurita, M. Burgos, R. Jiménez, Dev. Biol. 268, 39-52 (2004)

13. F. D. Carmona et al., J. Exp. Zool. 310B, 259-266 (2008).

14. J. Haeck, Neth. J. Zool. 19, 145-248 (1969).

15. C. J. Douady et al., Mol. Phylogenet. Evol. 25, 200-209 (2002).

16. Q. Wang, Y. Lan, E.-S. Cho, K. M. Maltby, R. Jiang, Dev. Biol. 288, 582-594 (2005)

17. C. Berthet, E. Aleem, V. Coppola, L. Tessarollo, P. Kaldis, Curr. Biol. 13, 1775-1785 (2003).

18. I. Hanukoglu, J. Steroid Biochem. Mol. Biol. 43, 779-804 (1992).

19. K. Suryamohan, M. S. Halfon, WIREs Dev. Biol. 4, 59-84 (2015).

20. J. Bowles et al., Dev. Cell 19, 440-449 (2010).

21. F. Zurita et al., Sex Dev. 1, 66-76 (2007)

22. J. S. Colvin, A. C. White, S. J. Pratt, D. M. Ornitz, Development 128, 2095-2106 (2001).

23. W. Bi et al., Proc. Natl. Acad. Sci. U.S.A. 98, 6698-6703 (2001).

24. N. H. Lazar et al., Genome Res. 28, 983-997 (2018).

25. A. J. Will et al., Nat. Genet. 49, 1539-1545 (2017).

\section{ACKNOWLEDGMENTS}

We thank A. Stiege, N. Brieske, U. Fisher, K. Macura, J. Fiedler K. Zill, C. Franke, and G. Mastrobuoni for technical support.
We thank P. Koopman for providing us with the Wt1-BAC vector. We thank the sequencing and genotyping facility of the Max Planck Institute of Molecular Cell Biology and Genetics in Dresden for helping with the initial long-read sequencing project. We thank the Animal Outcome Core Facility of the NeuroCure Center, Charité University, Berlin, for helping with strength tests. We also thank members of the Lupiáñez and Mundlos labs for fruitful discussions. Funding: This research was supported by grants from the Deutsche Forschungsgemeinschaft (grant numbers MU 880/15-1 and MU 880/16-1) and Max Planck Society. D.G.L. was supported by the Fundación Alfonso Martín Escudero and by a Helmholtz ERC Recognition Award grant from the Helmholtz-Gemeinschaft (ERC-RA-0033). Work conducted at the E. O. Lawrence Berkeley National Laboratory was performed under Department of Energy contract DE-AC02-05CH11231, University of California. A.V. and M.O. were supported by NIH grant R01HG003988. M.O. was supported by Swiss National Science Foundation grant PCEFP3_186993. O.S. was supported by the Austrian Science Fund FWF grant P32190. Author contributions: F.M.R., S.M., and D.G.L. designed the experimental approach. F.M.R., D.G.L., F.B., and R.J. captured moles and prepared the samples. F.M.R., M.S., and D.G.L. processed the samples for DNA, Hi-C, RNA, ChIP, and ATAC sequencing. S.A.H., B.T., A.M., and M.V. supervised bioinformatic analyses. R.S., D.H., M.-H.M., and H.K. assembled the genome. P.F., P.X., and O.S. performed the gene annotation and posterior genomic analyses. S.A.H. and A.B. analyzed the RNA-seq data. S.A.H. performed the epigenetic analysis and the prediction of synteny breaks. M.H. performed multispecies alignments. R.S., V.H., and T.K. analyzed chromatin interaction datasets. M.F.H. and S.A.W. performed hormone measurements. F.M.R., D.G.L., and A.H. performed the CYP17A1 enhancer transgenic experiment. F.M.R., I.H., and M.O. performed the FGF9 transgenic experiments. L.W. derived the XX mESC line and performed blastocyst aggregations. F.M.R., M.S.

S.M., and D.G.L. performed ELISA experiments and grip-strength tests. F.M.R., D.K.N.D., A.V., A.M., S.M., and D.G.L. participated in data interpretation and discussion. F.M.R., S.M., and D.G.L. wrote the manuscript with contributions from all other authors. Competing interests: The authors declare no competing interests. Data and materials availability: The Whole Genome Shotgun project has been deposited at DDBJ/ENA/GenBank under the accession number PRJNA494291. All transcriptomes and epigenetic data have been deposited in GEO under accession number GSE120589. Alignments have been deposited at https://bds.mpi-cbg.de/hillerlab/lberianMole.

\section{SUPPLEMENTARY MATERIALS}

science.sciencemag.org/content/370/6513/208/suppl/DC1 Materials and Methods

Supplementary Text

Figs. S1 to S20

Tables $\mathrm{S} 1$ to $\mathrm{S} 8$

References (26-100)

MDAR Reproducibility Checklist

Data S1 to S10

View/request a protocol for this paper from Bio-protocol.

27 August 2019; resubmitted 19 April 2020

Accepted 17 August 2020

$10.1126 /$ science aaz 2582 


\section{Science}

\section{The mole genome reveals regulatory rearrangements associated with adaptive intersexuality}

Francisca M. Real, Stefan A. Haas, Paolo Franchini, Peiwen Xiong, Oleg Simakov, Heiner Kuhl, Robert Schöpflin, David Heller, M-Hossein Moeinzadeh, Verena Heinrich, Thomas Krannich, Annkatrin Bressin, Michaela F. Hartmann, Stefan A Wudy, Dina K. N. Dechmann, Alicia Hurtado, Francisco J. Barrionuevo, Magdalena Schindler, Izabela Harabula, Marco Osterwalder, Michael Hiller, Lars Wittler, Axel Visel, Bernd Timmermann, Axel Meyer, Martin Vingron, Rafael Jiménez, Stefan Mundlos and Darío G. Lupiáñez

Science 370 (6513), 208-214.

DOI: $10.1126 /$ science.aaz2582

\section{Intersexuality in female moles}

Female moles are intersexual and develop masculinizing ovotestes, a distinctive trait among mammals. Real et al. investigated the origin of this trait by sequencing the lberian mole genome and applying comparative strategies that integrate transcriptomic, epigenetic, and chromatin interaction data. They identified mole-specific genomic rearrangements that alter the three-dimensional regulatory landscape of the androgen-converting gene CYP17A1 and the pro-testicular factor gene FGF9, both of which show distinct expression patterns in mole gonads. The use of transgenic mice confirms the capability of these factors to increase circulating testosterone levels and to induce gonadal masculinization. This study highlights how integrative approaches can reveal the phenotypic impact of genomic variation. Science, this issue p. 208

ARTICLE TOOLS

SUPPLEMENTARY

MATERIALS

REFERENCES

PERMISSIONS http://science.sciencemag.org/content/370/6513/208

http://science.sciencemag.org/content/suppl/2020/10/07/370.6513.208.DC1

This article cites 98 articles, 16 of which you can access for free http://science.sciencemag.org/content/370/6513/208\#BIBL

http://www.sciencemag.org/help/reprints-and-permissions

Use of this article is subject to the Terms of Service

Science (print ISSN 0036-8075; online ISSN 1095-9203) is published by the American Association for the Advancement of Science, 1200 New York Avenue NW, Washington, DC 20005. The title Science is a registered trademark of AAAS.

Copyright (C) 2020 The Authors, some rights reserved; exclusive licensee American Association for the Advancement of Science. No claim to original U.S. Government Works 